\title{
OPINIONES SOBRE CURRÍCULO: ENTRE LA FORMACIÓN EL DESEMPEÑO PROFESIONAL
}

Nora Agnes Vega Villalobos *

Al dar una mirada retrospectiva a la formación como enfermera a la luz de las corrientes filosóficas educativas encuentro alguna respuesta al porqué del desempeño profesional en mi país.

He tomado como puntos de referencia para el análisis de esta formación, aspectos sobresalientes del que hacer de enfermería como el planeamiento de la atención, distribución del trabajo, educación, investigados, relaciones humanas, trabajo interdisciplinario, evaluación por los clientes.

Mi primer año en la escuela la formación fue con tendencia tradicional como lo dice (LIBANEO, 1984; p. 23): "La actuación de la escuela consiste en la preparación intelectual y moral de los alumnos para asumir su posición en la sociedad". La disciplina era estricta, se controlaba la forma de vestir, no había libertad para comunicarse con los demás individuos. Por ejemplo al médico no se le podía preguntar nada, solo obedecer órdenes y sugerir respetuosamente cambios en las órdenes si considerábamos algo errado.

Desde el primer año y durante los tres años de formación los cursos eran teóricos prácticos. El saber se daba de modo que se tornara asimilable en el espacio y tiempo estipulado. O sea el papel de la escuela fue el de producir individuos competentes para el mercado de trabajo, transmitiendo eficientemente, informaciones precisas, objetivas y rápidas.

Los contenidos desarrollados durante los tres años eran transmitidos por aulas magistrales casi sin ayudas audiovisuales. Los exámenes eran de memorización, tipo desarrollo, extensos y a veces cargados en relación a un tema.

Reflexión sobre el que hacer de enfermería: Hay algunos aspectos en los que quisiéramos saber si esa formación ha respondido al que hacer cotidiano. Iniciemos por el plan de atención.

Para un planeamiento efectivo y visible de la atención del cliente, puede decirse que el enfermero piensa y reflexiona sobre los acontecimientos de salud a partir de su experiencia de vida. Los planes de atención han que dado en el pensamiento, por lo tanto son difíciles de discutir para determinar la continuidad en el cuidado. No se ha logrado desarrollar un instrumento práctico y accesible a los profesionales que permita evaluación paralela y una corrección inmediata.

*Profesora Asociada, Escuela de Enfermería, Universidad de Costa Rica y post graduanda en Enfermería, UFSC 
En consecuencia no se ha establecido un diálogo para discutir la factibilidad de los planes de atención con carácter de denuncia verdadera, compromiso de cambio y de acción como lo indica (FREIRE, 1987; p. 78). En este planeamiento se vislumbra un currículo oculto de formación subordinado al modelo médico por carecer la profesión de una identidad propia en su lenguaje de identificación de situaciones críticas. La filosofía de educación marcaba un currículo oculto donde se manifestaba "una superioridad del contexto cultural de las clases dominantes en contraparte con la pobreza cultural del contexto en que viven las clases dominadas (SOARES, 1989; p. 13).

El lenguaje de la formación en la escuela era diferente por la naturaleza del trabajo, pero no se dejó claro que aunque muestra ser diferente del lenguaje socialmente prestigiado, no es inferior ni deficiente. Así la enfermera puede estar hablando de otros aspectos de la situación del cliente que influyen en su condición de salud, incluso con mayor conocimiento y profundidad pero en las relaciones de fuerza su lenguaje no es reconocido como lenguaje legítimo. Es una área que se debe investigar para encontrar soluciones pertinentes, otros marcos de referencia para discutir la naturaleza de su trabajo.

La distribución de trabajo en la que privan conceptos importantes como capacidad del funcionario y riesgo del cliente se sigue el paradigma técnico linear. O sea se distribuye por número de personal y de clientes porque todos están preparados para desempeñar funciones definidas en situaciones también definidas (DOMINGUEZ, 1986; p. 254). Se ignoran dos de los elementos fundamentales: el sujeto y el objeto. Estará el individuo encargado de cuidar al cliente como sujeto bien preparado o será objeto de una metodología de trabajo que elude sus condiciones emocionales, sociales e técnicas. Habrá tenido en su formación, efectiva relación teórico práctica por medio de rondas, pre clínica, post-clínica, revisión bibliográfica, presentación de casos para asumir el trabajo asignado.

Esta vivencia fue más fuerte en le curso de administración al ser patente el enfrentamiento con la realidad de trabajo. Había que demostrar competencia técnica, cognoscitiva sin modelos establecidos ni referencia teórica clara. Se trabajaba bajo la supervisión de las enfermeras de la unidad que por supuesto tenían la misma orientación educativa heredada de las profesoras. La evaluación era más por intuición y comentarios del personal sobre la satisfacción que manifestaban al encontrar que el estudiante estaba completamente formado y daba a su trabajo el toque de un profesional experimentado.

Aspectos fundamentales del que hacer del enfermero, aprendidos en la escuela se esfumaron: las notas de enfermería y los planes de atención. Las notas de enfermería se delegaban y los planes quedaban en el pensamiento por lo que al ser tácitos se intuían y no se discutían. Este es un aspecto ético del cuidado que se debe reformular; la necesidad de tener un eje orientador del trabajo de enfermería que sea conocido de todos tos implicados incluyendo el cliente y un informe que constate lo ocurrido.

La educación en salud es una actividad importante del enfermero en prevención primaria con promoción y fomento de la salud. A hora en el periodo de formación no se usó el diálogo, la palabra como "una denuncia del mundo, ya que no hay denuncia verdadera sin compromiso de transformación, ni este sin acción (FREIRE, 1988; p. 78). Será que su formación le permite utilizar los acontecimientos del diario vivir para la 
discusión y la reflexión con los clientes a fin de instrumentalizarlos para que modifiquen la sociedad y su estado de salud como lo indica (CAPORILINI, 1991; p.36) o (FREIRE, 1987; p.86).

¿Se logrará que el enfermero tenga conciencia de la necesidad de capacitarse eficientemente y cumplir con competencia su papel técnico político en la preparación de individuos, grupos y comunidades para que sean los auto gestores de su salud? Enseñarlos a aprender - aprender sobre su condición de salud es papel del enfermero.

La formación fue doxológica, dada por la opinión de los demás: saber médico, los libros. No tanto por la "sofía", experiencia de los profesores, y menos aún por "episteme": ciencia, esta solo se mencionaba como la madre de la profesión pero sin identificar claramente su modo de actuar o ser en sí misma.

En la práctica, las acciones de enfermería con el cliente se encaraban en miras a cumplir órdenes médicas con una actitud crítico reproductiva sin cambiar nada porque no era lícito si no había salido de la investigación clínica médica.

Al ser clara la valía de la investigación médica clínica, como determinante imposición en el trabajo, la enfermera que da como un profesional con un lenguaje restricto, que orienta para significados particularistas y que por consiguiente lleva a la adquisición de un código restricto.

Probablemente de ahí que las enfermeras no enfaticen el desarrollo de su capacidad para realizar las anotaciones de enfermería y mucho menos la publicación de artículos. No hay preocupación por la elaboración de las notas de enfermería a pesar de que representan la comunicación escrita del progreso del cliente y sirve para salvar responsabilidades en caso de error de un tratamiento, y para realizar auditorias sobre la calidad del cuidado. Las notas de enfermería se delegan y no se asume el compromiso que implica.

No ha quedado claro que no hay lenguaje o saber más complejo o más simple, más o menos lógico, todos son adecuados a las necesidades y características de la cultura a la que le sirven, y igualmente válidas como instrumento de comunicación social.

La angustia por la crítica del medio sobre lo que se anota se debe a la ignorancia de saber qué es inadmisible usar los criterios de cierto y errado en relación al uso de la lengua. Lo que se considera errado no es lingüísticamente mejor ni peor que lo que se considera cierto, es apenas aquello que difere de la norma de prestigio socialmente privilegiada.

Al hacer anotaciones o presentar situaciones de los clientes, la enfermera teme expresarse porque desconoce que las diferencias no son de lenguaje sino de comportamiento delante de la tarea propuesta en la situación del cliente.

Para los sociólogos del lenguaje el establecimiento del modelo médico en la comunicación es ejercer un poder de violencia simbólica, "pues disimulan la discriminación social, por la legitimización e imposición de la cultura y del lenguaje del grupo dominante, en detrimento de la cultura y del lenguaje de otros grupos y así garantizan la preservación de la estratificación social" (SOARES, 1989; p. 54).

Hay de fondo un temor en la comunicación escrita por ejemplo en las publicaciones, hay una relación de fuerza simbólica. La mejoría de los bienes simbólicos utilizados en enfermería provienen del saber médico. Por lo que en enfermería ha privado la creencia de que las relaciones de fuerza simbólica presentes en la comunicación lingüística definen quién puede hablar, a quién y cómo; atribuyen valor y poder al lenguaje de unos y desprestigio al lenguaje de otros; imponen silencio a unos y e! papel de portavoz a otros. 
Le correspondió a la escuela la transmisión de ese lenguaje legítimo, en la inculcación de un proceso formal de reglas explícitas. Hasta que este modelo no cambie, hasta que no se desmitifique no se creará una conciencia de la verdadera identidad de la enfermera. Por tanto la solución estaría en transformaciones de la estructura social como un todo.

A la escuela corresponde enseñar a los futuros profesionales para que adquieran "los instrumentos necesarios en la lucha contra la desigualdad en la distribución de esos privilegios" (SOARES, 1989; p. 73). Le corresponde no solo enseñar tareas técnicas sino también políticas que expresan un compromiso de lucha contra las discriminaciones y las desigualdades sociales. O sea investigar para cuidar, cuidar para enseñar, enseñar para investigar, investigar para enseñar, enseñar para cuidar y cuidar para investigar.

Las relaciones humanas como lo mencioné al principio se mantenían en forma de subordinación la formación fortalecía el trabajo bien hecho pero solo. No se podía trabajar en grupo ni con otro compañero para dar cuidado al cliente, aunque estuviese grave. Por supuesto no se discutía con otros profesionales lo que se realizada con el cliente. Por tanto la preparación ha estado mutilada, ya que la participación envuelve la satisfacción de necesidades como la interacción con los demás hombres, la auto expresión, el desenvolvimiento del pensamiento reflexivo, el poder de crear y recrear cosas, y la valorización de sí mismo por los otros.

Con el tipo de formación se manejó un currículo oculto de marginalidad "donde el acceso a los beneficios está desigualmente repartido" (DIAZ, 1986; p. 19). Ya que la verdadera participación en el equipo de salud no debe ser con una presencia pasiva, sino con una intervención activa en la construcción del cuidado, es decir en la toma de decisiones, la participación no se mide en cuánto se toma parte sino cómo se toma parte.

Al no formar conciencia, la escuela no fortalecía en el futuro profesional ese deseo de "intervenir en las luchas sociales, económicas y políticas de su tiempo" (DIAZ, 1989; p. 24). No se formaba para "expresar necesidades o demandas, defender intereses comunes, alcanzar determinados objetivos económicos, sociales o políticos, o influir de manera directa en los poderes públicos" (DIAZ, 1989; p. 26).

La participación en la toma de decisiones del cuidado del cliente se puede decir que era dirigida o manipulada pues el grado de control sobre las decisiones era apenas de información y se tenía acceso a las informaciones menos importantes. En la lucha como profesión no se ha dado la preparación necesaria, el control fue tomado por líderes innatos o intuitivos, pero la participación en las decisiones de las instituciones no se destacó nunca quizá por esa falta de conciencia de hacer valer el trabajo realizado. Se aplica la concepción de que solo se participa realmente cuando se está entre iguales. Y por supuesto la estructura de poder, en laque el médico representa la clase privilegiada asfixia toda tentativa de participación en las decisiones del cliente y en el desarrollo de enfermería como profesión.

A hora no se puede pretender que de la escuela partiera la iniciativa para la organización y participación a nivel nacional e institucional si no se ha preparado a las enfermeras para que pasen por el aprendizaje del micro participación.

Ni siquiera en el equipo de enfermería se organiza el trabajo para la participación, hay un aparente dominio de la clase privilegiada (las enfermeras) sobre el personal técnico y asistente de paciente. Una jerarquización que no prepara para la macro participación. 
Para que se dé el trabajo en equipo es indispensable la vivencia desde a formación porque sino siempre será un agregado que no tiene fuerza ni se comparte.

No se ha permitido la opinión de los clientes sobre el tipo de profesional que se precisa formar. En la relación con los clientes hay cierto abismo en el diálogo porque como puedo dialogar, si me siento participante de un grupo de hombres puros, dueños de la verdad y del saber, para quienes todos lo que están fuera son esa gente, o son nativos inferiores (FREIRE, 1987; p. 80).

Si el cliente no puede hablar o no es escuchado. ¿Como puede darse el cambio efectivo que haga progresar el conocimiento en la profesión?

La formación del profesional de enfermería tenía un currículo oculto de sumisión al profesional médico, y con rasgos de mujeres consagradas subordinadas a los que tienen el saber (COLLIERE, 1989; p. 68). Situación que se manifiesta en la elaboración del plan de atención.

Las notas de enfermería y los planes de atención aprendidos en la formación se esfumaron: las notas se delegaban y los planes de atención quedaban en el pensamiento, imposible por tanto de ser discutidos por el personal. Las acciones de enfermería se encaran en miras a cumplir órdenes médicas.

Al hacer anotaciones o presentar situaciones de los clientes, la enfermera teme expresarse porque desconoce que las diferencias son de comportamiento y no de lenguaje delante de la tarea propuesta en la situación del cliente.

La distribución de trabajo sigue el paradigma técnico linear, por número de personal y de clientes. Se ignora la formación del personal para asumir el trabajo asignado.

La preparación para la participación en el trabajo interdisciplinario ha sido mutilada. No hubo ni siquiera el aprendizaje del micro participación entre colegas, menos aún macro participación.

En consecuencia, no se preparó para el diálogo con los clientes y para la discusión sobre el tipo de profesional que se precisa formar.

\section{REFERENCIAS BIBLIOGRAFICAS}

01. CAPORALINI, M. B. A transmissão do conhecimento e o ensino noturno. Campinas: Papirus, 1991.

02. COLLIERE, M. F. Promover a vida. Damaia: Sindicato de enfermeros portugueses, 1989.

03. DIAZ, B.J. O que é participação. 6 ed. São Paulo: Brasiliense, 1989.

04. DOMINGUEZ, J. L. Interesses humanos e paradigmas curriculares. Rev. Bras. Est. Pedag., Brasília, v. 67, n. 146, p. 351-66, mai/ago 1986.

05. FREIRE, P. Pedagogía del oprimido. 17. ed. Rio de Janeiro: Paz e Terra, 1988. p. 77-103.

06. LIBANEO, J. C. Democratização da escola pública: a pedagogia crítico-social dos conteúdos. São Paulo: Loyola, 1984. p. 19-44.

07. SOARES, M. Linguagem e escola: uma perspectiva social. 7ed. São Paulo: Ática, 1989. 